\title{
Suicidal Intra-Oral Gunshot with Labial Laceration
}

\author{
Pierucci $\mathbf{G}^{\mathbf{1}}$, Toni $\mathrm{C}^{2 *}$ and Filomena $\mathrm{C}^{2}$ \\ ${ }^{1}$ Department of Legal Medicine, University of Pavia, Italy \\ ${ }^{2}$ Department of Legal Medicine, University of Pisa, Italy \\ *Corresponding author: Toni C, Department of Legal \\ Medicine, University of Pisa, Via Roma 55, Pisa, 56126, \\ Italy
}

Received: J une 05, 2017; Accepted: J une 23, 2017;

Published: July 07, 2017

\section{Abstract}

This paper describes a case of suicide using intra-oral gunshot $(7.65 \mathrm{~mm}$ gun), with the bullet going in through the throat, out through the nape, at an angle, from the bottom up and from front to back. In addition, there was also a non-ballistic injury, i.e. a laceration on the inside of the lower lip that dug a few millimeters into tiny, tight depressions, shaped like the tips of the lower incisors. In that area, the depressions were all broken and dislocated, as were the teeth. The lip injury, with a macroscopically lively appearance, did not show any additional marks of gunshot and had been caused by firm pressure in the oral cavity, due to detonation.

Such injuries must be distinguished from injuries caused by bullets when they are shot in: gunshot in or near the oral site can be, though rarely, suggestive of murder, and that's why such method must be clearly distinguished from suicide methods.

Keywords: Suicide; Gunshot; Depression

\section{Introduction}

Suicide using gunshot is a comparatively frequent event, especially in countries were gun laws are more relaxed, where such phenomenon has spread so much that such suicide method is now one of the most frequently observed [1].

In Italy, the highest number of deaths by gunshot happens in the south: a retrospective survey conducted at the Institute of Forensic Medicine of Bari (Puglia) from 1988 to 2003 found 717 deaths from gunshot injuries, 82 of which were suicides (11.5\%), with 81 men (98.7\%) and just one woman [2].

The site of self-inflicted wounds depends on the type of fire arm, the sex of the victim, and whether the victim is right- or left-handed [3]. The anatomic site that is most commonly shot at is the head, followed by the chest, then the abdomen [4].

As to the head, some authors find that the most frequently shot site is the right or left temple, depending on whether the victim is right- or left-handed [5], while others find it is the oral cavity [3]. The fact the victim's sex affects the choice of anatomic site has been confirmed by Cohle [6] who in his study found that $88.9 \%$ of men kill themselves by shooting at their heads, versus $48.4 \%$ of women.

Some rare cases of murder through shooting into the mouth at close range have been reported too. The main difference is that murders use a different path and cause both lip and dental injuries. Such injuries can also occur in suicide, like this one. So, injuries need to be carefully observed to tell the difference. That's why this is such an interesting case as an example of injuries that could fit in with methods, homicide and suicide.

\section{Case Presentation}

A 73-year-old man, a retired pharmacist who lived with his wife, suffering from depression, loath to see a doctor in self-treatment. Found one morning at 06:40 am by his wife, who, worried by the noise of gunshot coming from their bedroom, had rushed into the room. The body was lying down, in a "pool of blood", as described by his wife, perpendicular to then-suite door.

The A\&E medical staff alerted by the dead man's wife found no pulse in the carotid or radial artery, tried the usual resuscitation, and declared him dead.

Forensic police took the weapon he had shot with, which was a semiautomatic gun, made by TANFOGLIO Giuseppe mod. GT 30, $7.65 \mathrm{~mm}$ parabellum, legally held by the owner, and found a bullet in the barrel and the magazine fitted in. The weapon was in the washbasin of then-suite.

\section{Inspection of the body}

A widely blood-stained, $167 \mathrm{~cm}$ long male body.

Wearing: grey pyjamas, also widely blood-stained.

Treatment: oro-pharyngeal airway fitted in and electrodes in the usual and marks.

Minor bruising in both eye lids. Bleeding from both ears, more on the left. Partly clotted blood at the mouth and nose openings.

Warped tip of the head, extending on both sides to the occipital walls, with noticeable bone "step". Just right of the median, near the junction with the occipital wall, the scalp shows a star-shaped gap (approximately $4 \times 4 \mathrm{~cm}$ ), with blood-soaked edges, which goes deeper in, with a bone breach oozing pulpy, bloody brain matter (Figure 1).

After forcing the rimaorisopen, as it was shut tight by rigor mortis, the entire mucous issues look slightly smoked, more markedly on the mouth floor, especially at the back of the tongue and on the roof, mainly at the back, on the soft palate. A rounded gap can be observed here and is carefully probed with a probe. It catches on the top and back, and slightly on the right, at approximately 45 degrees, and then it comes out of the bone-integumentary breach on the occipital region (Figure 2). 


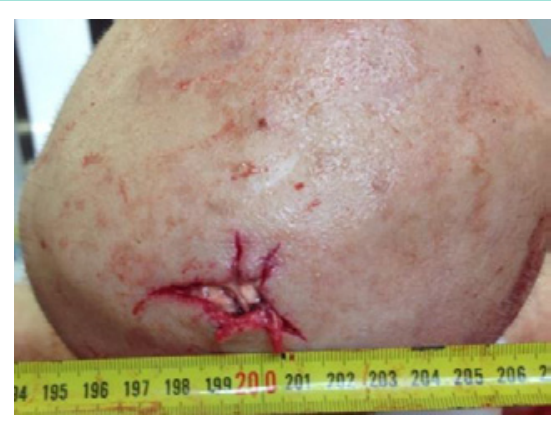

Figure 1: Bullet exit wound in occipital region.

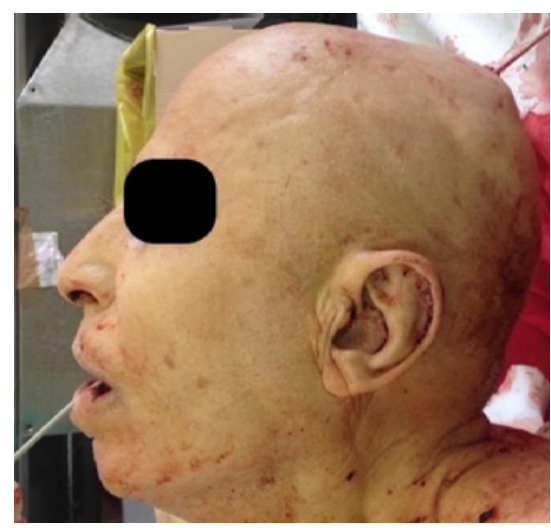

Figure 2: The probe indicates bullet path.

The mucous surface of the lower lip exhibits a compound bruised-torn injury, which looks like a "horseshoe", with the longer axis going left and slightly down, to meet the convex part of the figure. Such injury is more or less aligned with the median, and its overall dimensions are $15 \mathrm{~mm}$ (crosswise) x $14 \mathrm{~mm}$ (vertical); the upper branch (the lower one, in the Figure) reaches almost as far as the margin of the lip. The perimeter of the injury breaks up into an unevenly-edged, wide groove, whose depth is uneven but deeper in some depressions, especially on the upper branch, which dugs into the muscular layers. Two of them are coupled near the median, one on the right and one on the left, both shaped like rectangles. Another depression is found on the convex part of the groove; other, more blurred and superficial recesses are located in the lower branch. The part of the mucous membrane that is surrounded by the groove and joined to the residual mucous tissue on the right side is extensively soaked with blood and purplish in color. Other tiny bruised-grazed injuries, a sort of cracks, instantly appear on the right of the main injury (Figure 3).

The lower median incisors are protruding, loosely held by their sockets, due to the fracture of the front lamina of the alveolar wall (Figure 4).

\section{Discussion}

In this case, a post-mortem was prohibited by the legal authorities, so these comments and conclusions are incomplete. However, some facts look interest in gland comparable with the literature.

As everyone knows, most gunshots through the mouth are suicidal

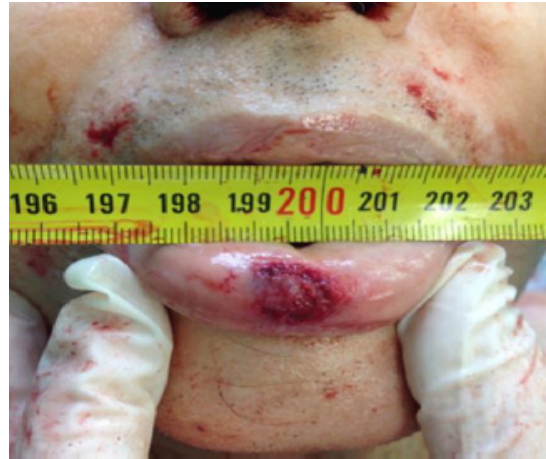

Figure 3: Labial laceration. The figure is reversed by overthrow of lower limb.

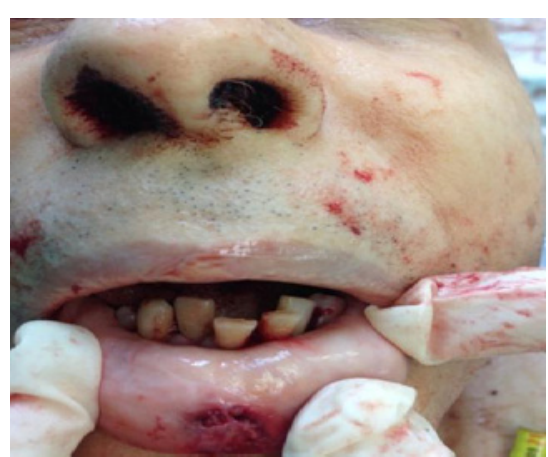

Figure 4: Labial laceration with corresponding dental luxation.

and actually account for a high proportion of them [7-9]. Therefore, such injuries hardly ever raise the question of distinguishing suicidal from homicidal forms. However, there have been murders in which the bullet was shot through the victim's mouth with or without per oral injuries, so in these cases the injuries and circumstances need to be thoroughly investigated to tell the difference. In this specific case, the death scene was not examined by the forensic doctor, so the diagnosis only relied on the out ward examination of the body.

Murders using gunshot through the oral cavity mainly cause injuries in or near the surrounding sites [10,11]. After all, injuries in surrounding sites appear quite frequently in suicide as well $[3,12,13]$. In this case, the lips were most affected.

Moreover, other factors, especially the path, were taken into account to establish the nature of the event [14]. In murder, the path is generally horizontal, it touches the oral cavity at the back and can also involve the tongue $[4,8]$. In suicide, the path is usually at an angle, from the bottom up and from front to back, the bullet is shot more frontally, close to the palate, and flies out through the nape: as in this case [4].

In addition, in suicide, additional marks of discharge can be found in the oral cavity in the form of widespread smoking, as well as at the edges of the injury, and maybe a few traces very close by (known as explosive effects; etc.). In homicidal forms, the gun's muzzle is hardly ever put into the oral cavity; in most cases, it is far from the "target". In this case, per oral or even dental injuries can be easily observed. The distinction between the two forms becomes, instead, more complicated when the lips are involved. After all, 
such site of the body is often injured in suicide [15]. Such injuries come in many different shape sand are usually caused by the lower teeth, mainly the lower incisors, dislocated forward by the fracture of the alveolar processes in the area. This phenomenon is of course the result of a sharp increase in intraoral pressure, as the mouth is a half-closed cavity. The distinctive morphological features of each case may depend on many different factors: ballistic (type of weapon and ammunition, exact location of muzzle; etc.) and anatomical (shape of oral cavity; state of teeth; tightness of lips; etc.).

A differential diagnosis of lower lip injuries may have to be performed to make a distinction between injuries from gunshot and injuries caused by a person who attacks and gags the victim with the hands. In this case, apart from the general picture, lower lip injuries from external pressure are usually much less marked, and the edges of the teeth often leave a mark, maybe a super facial laceration, not the deep lacerations we observed in this case. What's most remarkable about this case is that the tips of the teeth cut in and left a deep and very noticeable mark, a sort of cross-section of the teeth: which means that the penetration was almost perpendicular, due to the dislocation? A person who tried to choke someone to death would hardly ever dislocate the victim's teeth, and, if that happened, then it would happen in a front-to-back direction.

In this case, the lip injury had a distinctive shape, a sort of horseshoe that is suggestive of an inward bite. No similar cases have been found in the texts we consulted.

The double row of lip injuries, the upper and the lower one, probably comes from the dislocated teeth pushing hard against the mucous surface of the lower lip, in two steps.

\section{References}

1. Lester $D$. The availability of firearms and the use of firearms for suicide: a study of 20 countries: ActaPsychiatr. Scand. 1990; 81: 146-147.
2. Solarino EM, Vella ND. Fatal firearm wounds: A retrospective study in Bari (Italy) between 1988 and 2003; Forens. Sci. Int. 168: 95-101.

3. Di Maio MJ. Gunshot Wounds. Practical Aspects of Balistics and Forensic Techniques, Elsevier, New York, 1985

4. Avis SP. Suicidal gunshot wounds. 1994; Forens Sci Int. 67: 41-47.

5. Zietlow C, Hawley DA. Unexpectedly Homicide - Three intraoral gunshot wounds.1993; Am J Forensic Med Pathol. 14: 230-233.

6. Cohle. Handgun suicides. 1977; Forensic Sci. Gaz.; in V.M.J Di Maio, 294295.

7. Stone C. Characteristics of Firearms and Gunshot Wounds as Markers of Suicide. Am J Forens Med Path. 1992; 13: 275-280.

8. Azmak D, Altun G, Koc S, Yorulmaz C, Ozaslan A. Intra- and perioral shooting fatalities. Forens Sci Int. 1999; 101: 217-227.

9. Desinan L, Mazzolo GM. Gunshot fatalities. Suicide, homicide or accident? A series of 48 cases. Forens Sci Int. 2005; 147S: S37-S40.

10. Suwanjutha T. Direction site and the muzzle target distance of bullet in the head and neck at close range as an indication of suicide or homicide. Forens Sci Int. 1998; 37: 223-229.

11. Fatteh A. Homicidal Gunshot Wound of Mouth. J Forens Sci Soc. 1972; 12: 347-349.

12. Eisele W, Reay DT, Cook A. Sites of suicidal gunshot wounds. J Forens Sci. 1981; 26: 480-485.

13. Lee KA, Opeskin K. Gunshot suicide with nasal entry. Forens Sci Int. 1995; 71: 25-31.

14. Druid $\mathrm{H}$. Site of entrance wound and direction of bullet path in firearms fatalities as indicators of homicide versus suicide. Forens Sci Int. 1997; 88: 147-162.

15. Perdekamp G, Nadjem H, Merkel J, Braunwarth R, Pollak S, Thierauf A. Two-gun suicide by simultaneous shots to the head: interdisciplinary reconstruction on the basis of scene investigation, autopsy findings, GSR analysis and examination of firearms, bullets and cartridge cases. Int $\mathrm{J}$ Legal Med. 2011; 479-485.
Austin J Forensic Sci Criminol - Volume 4 Issue 2 - 2017 ISSN : 2380-0801 | www.austinpublishing group.com Toni et al. () All rights are reserved
Citation: Pierucci G, Toni C and Filomena C. Suicidal Intra-Oral Gunshot with Labial Laceration. Austin J Forensic Sci Criminol. 2017; 4(2): 1064. 